\title{
¿Influyen las barreras arquitectónicas en la ayuda personal recibida en los desplazamientos de la persona dependiente?
}

\author{
Do the architectural barriers affect the daily personal help received by the dependent \\ person in displacements?
}

I. de Rosende, S. Santos

\begin{abstract}
Resumen
Introducción. Diversos estudios demostraron una asociación significativa entre las barreras arquitectónicas y el desempeño de las actividades diarias con ayuda personal, en sujetos mayores o con discapacidad física. No se localizaron investigaciones similares en individuos dependientes.

Objetivo. El objetivo principal de este estudio fue determinar la relación entre las barreras arquitectónicas y la ayuda recibida en los desplazamientos dentro del hogar del sujeto dependiente. Como objetivo secundario se planteó identificar diversos factores relacionados con el apoyo en la actividad reseñada.

Material y métodos. Se revisaron los Baremos de Valoración de la Dependencia aplicados por el investigador, como técnico valorador, durante 16 semanas consecutivas, en 7 Ayuntamientos de A Coruña, en los que se reconoció dependencia a solicitantes mayores de edad.

Se estudió la relación entre el apoyo personal recibido en los desplazamientos y la edad, género, condiciones de salud, comorbilidad, hospitalización y diversas barreras arquitectónicas. Se construyó un modelo de remisión logística para identificar los factores predictores de la ayuda en la actividad, incluyendo las variables significativas del análisis bivariante.

Resultados. Se analizaron 208 baremos (68,3\% mujeres; mediana 84 años). En el modelo, 3 condiciones de salud (deterioro cognitivo y/o demencia, accidente cerebrovascular y enfermedad pulmonar obstructiva crónica), la hospitalización y 2 barreras (peldaño y puerta estrecha) se asociaron significativamente con la ayuda personal recibida en los desplazamientos.

Conclusión. La dependencia en los desplazamientos se explica por varias condiciones de salud, la hospitalización y diversas barreras. Se confirma la influencia del entorno físico sobre el desempeño diario, por lo que es necesario promover el asesoramiento en adaptaciones del hogar.
\end{abstract}

\footnotetext{
Abstract

Introduction. Several studies have shown a significant association between architectural barriers and performance of daily activities with personal assistance in elderly subjects or those with physical disabilities. No similar investigations were found regarding dependent persons.

Objective. Principal objective: To determine the relationship between the presence of architectural barriers and personal assistance received in displacements within the home of dependent subject. Secondary objective: To identify various factors related to the support in the mentioned activity.

Material and methods. We reviewed the Dependency Evaluation Scale applied by the researcher as an evaluation technique during 16 consecutive weeks in 7 municipalities of La Coruña in which dependency was admitted in elderly applicants.

The relationship between the personal help received in displacements and age, gender, several health conditions, comorbidity, hospitalization and different architectural barriers was studied. A logistic regression model was constructed to identify predictors of personal support in the activity, including significant variables in the bivariate analysis.

Results. A total of 208 scales (68.3\% female; median 84 years) were analyzed. In the model, three health conditions (cognitive deterioration and/or dementia, stroke, chronic obstructive pulmonary disease), hospitalization and two barriers (step and narrow door) were significantly associated with the personal assistance received in the displacements.

Conclusion. The dependence in the displacements is explained by several health conditions, hospitalization and various barriers. This confirms the influence of the physical environment on daily performance, so that education on home adaptations is necessary.
} 
Palabras clave

Caminata; Cuidadores; Dependencia (psicología); Estructuras de acceso; Vivienda

Keywords

Architectural accessibility; Caregivers; Dependency (psychology); Housing; Walking

\section{Introducción}

El sistema español de protección a la dependencia persigue el objetivo de mantener el individuo en el entorno en el que desarrolla su vida, con la mayor autonomía posible. Permanecer en el domicilio es una elección prioritaria en la población. Sin embargo, la atención en el hogar puede ocasionar limitaciones en la salud del cuidador y experiencias de «sobrecarga». La independencia en el desempeño diario disminuye y retrasa la inversión en servicios de apoyo domiciliario y en residencias ${ }^{1}$. Las evidencias reseñadas previamente sugieren analizar qué estrategias favorecen de forma simultánea el mantenimiento en el hogar y la realización de las actividades con la menor ayuda posible.

El modelo social de la discapacidad, reconocido por la Organización Mundial de la Salud (OMS), en la Clasificación Internacional del Funcionamiento, la Discapacidad y la Salud (CIF) del año 2001 ${ }^{2}$, destaca la existencia de factores en el contexto que influyen en la salud y el funcionamiento. Un ejemplo son las barreras arquitectónicas. El presente trabajo analiza la influencia ejercida por el entorno físico domiciliario sobre la probabilidad de recibir ayuda personal en los desplazamientos dentro del hogar del individuo dependiente. Existen pocos estudios acerca de la relación entre la presencia de barreras y el apoyo de un cuidador en las actividades diarias. Se realizaron en muestras de sujetos mayores ${ }^{3}$, usuarios de silla de ruedas ${ }^{4}$ y adultos con discapacidad física ${ }^{5}$ and 6 . No se han encontrado investigaciones similares dirigidas a individuos con un grado moderado o superior de dependencia, titulares de los derechos reconocidos en el sistema público español de protección.

El objetivo principal consiste en determinar la posible asociación entre la existencia de barreras y el apoyo de otra persona en los desplazamientos dentro del hogar. Como objetivo secundario, el estudio se dirige a identificar diversos factores relacionados con la ayuda en la actividad reseñada.

\section{Material y métodos}

Se trata de un estudio observacional y transversal, realizado en el Servicio de Dependencia y Autonomía Personal de A Coruña (Xunta de Galicia). En esta administración, uno de los investigadores desempeña la profesión de técnico valorador, consistente en la aplicación del Baremo de Valoración de la Dependencia (BVD) ${ }^{7}$, instrumento oficial para evaluar la dependencia.

El estudio consiste en una revisión de todos los $\mathrm{BVD}^{7}$ aplicados por uno de los investigadores, como valorador de dependencia, durante 16 semanas consecutivas, que cumplen con los criterios de inclusión siguientes:

- El solicitante de reconocimiento de la dependencia es mayor de edad.

- El BVD ${ }^{7}$ se aplicó en el domicilio, en uno de los siguientes ayuntamientos limítrofes: A Coruña, Abegondo, Bergondo, Cambre, Carral, Oleiros y Sada.

- En la entrevista participó el solicitante sin trastorno cognitivo y/o el cuidador informal mayor de edad. Presenta un trastorno cognitivo cuando los informes recogen el diagnóstico de deterioro cognitivo, demencia, síndrome amnésico orgánico o retraso mental y/o cuando obtiene 3 o más puntos en la versión española del test de Pfeiffer $^{8}$, en sujetos que leen y escriben, o un mínimo de 4 puntos en los restantes participantes. El término «informal» hace referencia a la ausencia de una retribución económica por el cuidado.

- En la semana previa, el solicitante accedió a una estancia diferente de la habitación para desempeñar alguna de las actividades de autocuidado.

- El BVD ${ }^{7}$ reconoció un grado I (moderado), II (severo) o III de dependencia.

Se excluyeron los $\mathrm{BVD}^{7}$ aplicados a menores de 65 años, con una enfermedad mental como causa principal de la dependencia, que realizaban sin ayuda física las transferencias corporales, los desplazamientos dentro del hogar vinculados al autocuidado y/o el acceso al exterior. 
La variable de estudio (dependiente) consiste en la evaluación de la ayuda personal recibida en los desplazamientos dentro del hogar vinculados al autocuidado, tarea definida en el $\mathrm{BVD}^{7}$. Se valora a través de 2 categorías: recibe ayuda de otra persona (supervisión y/o apoyo físico) vs. no recibe ayuda.

Como posibles variables explicativas (independientes) de la ayuda personal recibida en los desplazamientos, se analizaron 2 grupos: relativas a la persona en situación de dependencia y la existencia de barreras arquitectónicas para el sujeto dependiente. En el primer grupo se incluye el género; edad; comorbilidad, a través de la versión abreviada del índice de Charlson ${ }^{9}$ and ${ }^{10}$; así como la hospitalización en los 2 últimos años (sí vs. no). También se estudió la presencia de 12 condiciones de salud detalladas en la tabla 1 en los informes clínicos aportados con la solicitud (sí vs. no).

Tabla 1. Características de las personas en situación de dependencia. Participantes $(\mathrm{n}=208)$

\begin{tabular}{|c|c|}
\hline Características & Resultados \\
\hline Mujer $^{\mathrm{a}}$ & 68,3 \\
\hline Edad & 84 \\
\hline Mediana & 59 y 100 \\
\hline Mínimo y máximo & 80 y 89 \\
\hline Percentiles 25 y 75 & 85 \\
\hline Mujer: mediana & 82 \\
\hline Varón: mediana & 49,5 \\
\hline Condiciones de salud ${ }^{\mathrm{a}}$ & 42,4 \\
\hline Deterioro cognitivo y/o demencia & 21,2 \\
\hline Poliartrosis & 17,3 \\
\hline Accidente cerebrovascular & 15,4 \\
\hline Depresión o síndrome ansioso-depresivo & 13,5 \\
\hline Enfermedad pulmonar obstructiva crónica & 12,5 \\
\hline Tumor & 11,1 \\
\hline Insuficiencia cardiaca & 9,1 \\
\hline Enfermedad de Parkinson o parkinsonismo & 8,7 \\
\hline Coxartrosis y/o gonartrosis & 1,9 \\
\hline Fractura de fémur o luxación de cadera & 1,4 \\
\hline \multicolumn{2}{|l|}{ Ceguera } \\
\hline \multirow[t]{2}{*}{ Amputación de miembro inferior } & 1 \\
\hline & 0 y 5 \\
\hline Comorbilidad: índice de Charlson abreviado & 1 у 2 \\
\hline \multicolumn{2}{|l|}{ Mediana } \\
\hline Mínimo y máximo & 48,6 \\
\hline \multicolumn{2}{|l|}{ Percentiles 25 y 75} \\
\hline Hospitalización en los 2 últimos años ${ }^{a}$ & \\
\hline
\end{tabular}

${ }^{\text {a }}$ Resultados expresados en porcentaje.

Otra de las variables independientes consiste en la valoración de la existencia en el hogar de barreras arquitectónicas para la persona dependiente, mediante el instrumento Housing Enabler Screening Tool ${ }^{11,}$ 12 and 13 . Publicado por Iwarsson y Slaug (2010), define la accesibilidad como una relación de ajuste y equilibrio entre las capacidades del sujeto y el contexto físico. Comprende 2 componentes («personal» $\mathrm{y}$ «ambiental») y se evalúa mediante la integración de ambos, al establecer que las características del hogar no suponen un impedimento de por sí, sino que causan diferentes magnitudes de «problemas de accesibilidad» dependiendo de las limitaciones de cada individuo ${ }^{11,12 \text { and } 13}$.

En primer lugar se analiza el componente «personal», determinando qué «limitaciones funcionales» presenta el individuo, definidas como restricciones en la habilidad para desarrollar acciones físicas y 
mentales fundamentales en la vida diaria. También se evalúa si usa silla de ruedas u otros productos de apoyo para la movilidad. A continuación, se valora la existencia en el domicilio de un conjunto de 60 características del contexto físico (componente «ambiental»). Por último, con respecto a cada una de las características anteriores presentes, se determina si suponen o no un «problema de accesibilidad» para la persona, atendiendo a su perfil de «limitaciones funcionales» y/o a los dispositivos que utiliza en la movilidad $^{11,12 \text { and } 13}$. Por ejemplo, las puertas o pasillos estrechos solo se consideran un impedimento para los usuarios de silla de ruedas o de otros dispositivos para la deambulación.

Del total de ítems del componente «ambiental», en el presente estudio se incluyen 4 características del hogar recogidas en la tabla 2. Dos traductores profesionales bilingües realizaron una adaptación al castellano de los ítems seleccionados. El primero de ellos llevó a cabo la traducción del inglés al castellano; el segundo, la retrotraducción castellano-inglés. Ambos analizaron las 2 versiones en un proceso de discusión con los autores del presente estudio, concluyendo que existe una equivalencia de contenido entre la traducción y la escala original. El Housing Enabler Screening Tool 11, 12 and 13 recomienda adaptar la definición de las barreras a la legislación vigente en el país en el que se desee utilizar. Por ello, esta escala se comparó con la norma gallega de accesibilidad ${ }^{14}$, modificándose 3 ítems señalados en la tabla 2.

Tabla 2. Barreras arquitectónicas para la persona en situación de dependencia, en los desplazamientos dentro del hogar vinculados al autocuidado. Participantes $(n=208)$

\begin{tabular}{ll}
\hline Barreras arquitectónicas & $\begin{array}{l}\text { Personas que encuentran la barrera en } \\
\text { los desplazamientos estudiados }\end{array}$ \\
\hline
\end{tabular}

Puerta estrecha (menos de $80 \mathrm{~cm}$ de espacio libre) ${ }^{\mathrm{a}} 63$

Pasillo estrecho (menos de $1,2 \mathrm{~m})^{\mathrm{a}} \quad 53,8$

Peldaño, umbral, diferencia de nivel entre $\quad 7,2$

habitaciones/espacios ${ }^{\mathrm{a}}$

Escaleras (no hay ascensor) $\quad 3,4$

${ }^{\text {a }}$ Barrera cuyo enunciado ha sido modificado para cumplir con la normativa vigente.

${ }^{\mathrm{b}}$ Resultados expresados en porcentaje.

El investigador principal evaluó las 4 características del hogar seleccionadas, a través de 2 categorías: se encuentra presente vs. ausente. Con respecto a cada una de las existentes en el domicilio, formuló la siguiente cuestión: según el Housing Enabler Screening Tool ${ }^{11,12}$ and 13, la característica presente ¿supone un «problema de accesibilidad» para la persona dependiente? Las respuestas son «sí» o «no»; si es afirmativa, en el presente estudio se considera que existe una barrera arquitectónica para el participante.

En cuanto al análisis de datos, las variables cualitativas se describen mediante porcentajes. Para las cuantitativas, al comprobar que no cumplen la hipótesis de normalidad, según el test de KolmogorovSmirnov, se utilizó la mediana, el valor mínimo, máximo y los percentiles 25 y 75 . Para estudiar la relación entre las variables independientes y el apoyo personal recibido se empleó el $\chi^{2}$ de Pearson o el test exacto de Fisher con las variables cualitativas, y la t de Student para la comparación de medias. Con el fin de conocer los factores predictores de recibir ayuda personal en los desplazamientos interiores, se construyó un modelo de remisión logística, incluyendo las variables significativas en el análisis bivariado mediante el método por pasos hacia atrás (Wald). Se calcularon las odds ratio (OR) de recibir ayuda personal y los intervalos de confianza del 95\% (IC 95\%). El nivel de significación empleado fue p $<0,05$ (bilateral) y se utilizó el programa SPSS ${ }^{\circledR}$ v.17.0.

La información analizada procede de copias de BVD aportadas por la administración, mediante disociación previa de los datos de identificación. El estudio fue aprobado por el Comité Ético de Investigación Clínica de Galicia. 


\section{Resultados}

El investigador aplicó $297 \mathrm{BVD}^{7}$ durante el periodo analizado. El 70\% (n = 208) cumplía los criterios de inclusión del estudio. El perfil de persona dependiente se caracterizó por el predominio del género femenino y una edad próxima a los 85 años, mayor en las mujeres. El diagnóstico más frecuente fue el deterioro cognitivo y/o la demencia $(49,5 \%)$. Se obtuvo una mediana de un punto en el índice de Charlson $^{9 \text { and } 10}$, reflejando ausencia de comorbilidad. En los 2 últimos años, casi la mitad tuvo un ingreso hospitalario (tabla 1). El 57,7\% de los sujetos dependientes desempeñaban los desplazamientos dentro del hogar vinculados al autocuidado con ayuda personal. La tabla 2 detalla el porcentaje de participantes que encontró cada barrera en los desplazamientos estudiados. La más frecuente fue la existencia de puertas de ancho inadecuado (63\%).

Recibir ayuda personal en los desplazamientos analizados es significativamente más frecuente en los participantes con deterioro cognitivo y/o demencia $(p=0,002)$ o con accidente cerebrovascular $(\mathrm{p}=0,002)$; sucede lo contrario en los sujetos con enfermedad pulmonar obstructiva crónica $(\mathrm{p}=0,003)$. La comorbilidad se asocia de forma estadísticamente significativa con el apoyo personal en la actividad $(\mathrm{p}=0,008)$, al igual que la hospitalización en los 2 últimos años $(\mathrm{p}=0,029)$ (tabla 3). Realizar los desplazamientos interiores con ayuda es significativamente más frecuente en los participantes que encuentran las siguientes barreras arquitectónicas: puertas de ancho inadecuado $(\mathrm{p}=0,021)$, pasillo estrecho $(\mathrm{p}=0,005)$, así como peldaños, umbrales o diferencias de nivel $(\mathrm{p}=0,002)$ (tabla 4$)$.

Tabla 3. Relación entre las características de la persona dependiente y la ayuda personal en los desplazamientos dentro del hogar vinculados al autocuidado. Participantes $(n=208)$

Características de la persona dependiente

¿Desempeña la actividad con ayuda de otra persona?

\begin{tabular}{|c|c|c|c|}
\hline & Sí (n = 89) & No & Valor de $\mathrm{p}$ \\
\hline Mujer & 69,7 & 67,2 & 0,709 \\
\hline Edad: media $(D E)$ & $83,66(8,45)$ & $83,76(6,52)$ & 0,925 \\
\hline \multicolumn{4}{|l|}{ Condiciones de salud } \\
\hline Deterioro cognitivo y/o demencia: sí & 61,8 & 40,3 & $0,002^{\mathrm{a}}$ \\
\hline Poliartrosis: sí & 42,7 & 42 & 0,922 \\
\hline Accidente cerebrovascular: sí & 31,5 & 13,4 & $0,002^{\mathrm{a}}$ \\
\hline Depresión o síndrome ansioso-depresivo: sí & 18,5 & 15,7 & 0,603 \\
\hline Enfermedad pulmonar obstructiva crónica: sí & 6,7 & 21,8 & $0,003^{\mathrm{a}}$ \\
\hline Tumor: sí & 16,9 & 10,9 & 0,215 \\
\hline Insuficiencia cardíaca: sí & 13,5 & 11,8 & 0,711 \\
\hline Parkinson o parkinsonismo: sí & 12,4 & 10,1 & 0,605 \\
\hline Coxartrosis y/o gonartrosis: sí & 6,7 & 10,9 & 0,3 \\
\hline Fractura de fémur o luxación de cadera: sí & 9 & 8,4 & 0,882 \\
\hline Ceguera: sí & 2,2 & 1,7 & 1 \\
\hline Amputación de miembro inferior: sí & 2,2 & 0,8 & 0,577 \\
\hline Charlson abreviado: media $(D E)$ & $1,80(1,13)$ & $1,39(1,07)$ & $0,008^{\mathrm{a}}$ \\
\hline Hospitalización en los 2 últimos años: sí & 57,3 & 42 & $0,029^{\mathrm{a}}$ \\
\hline
\end{tabular}

Resultados expresados en porcentaje, a menos que se indique lo contrario. DE: desviación estándar.

${ }^{\mathrm{a}} \mathrm{p}<0,05$. 
Tabla 4. Relación entre la presencia de barreras arquitectónicas y la ayuda de otra persona en los desplazamientos dentro del hogar vinculados al autocuidado. Participantes $(n=208)$

La persona dependiente, ¿encuentra la barrera en los desplazamientos estudiados?

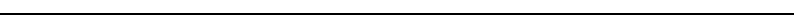

Puerta estrecha (menos de $80 \mathrm{~cm}$ de espacio libre): sí

Pasillo estrecho (menos de 1,2 m): sí

Peldaño, umbral, diferencia de nivel entre habitaciones/espacios: sí

Escaleras (no hay ascensor): sí
¿Desempeña la actividad con ayuda de otra persona?

\begin{tabular}{ccc}
\hline Sí $(\mathrm{n}=89)$ & No & Valor de p \\
\hline 71,9 & 56,3 & $0,021^{\mathrm{a}}$ \\
65,2 & 45,4 & $0,005^{\mathrm{a}}$ \\
13,5 & 2,5 & $0,002^{\mathrm{a}}$ \\
5,6 & 1,7 & 0,140
\end{tabular}

Resultados expresados en porcentaje.

${ }^{\mathrm{a}} \mathrm{p}<0,05$.

La tabla 5 detalla el modelo de remisión logística construido. Los factores asociados significativamente con la ayuda de otra persona en los desplazamientos estudiados son 3 condiciones de salud (deterioro cognitivo y/o demencia, ACV y EPOC), hospitalización y 2 barreras (peldaño, umbral o diferencia de nivel; puerta estrecha). La mayor OR de recibir ayuda personal se obtuvo con respecto a la existencia de escalones o umbrales $(\mathrm{OR}=12,26)$.

Tabla 5. Modelo de remisión logística para explicar la ayuda personal recibida en los desplazamientos dentro del hogar vinculados al autocuidado (recibe ayuda vs. no). Participantes $(n=208)$

\begin{tabular}{lccc}
\hline Variables explicativas incluidas en el modelo & OR & IC 95\% de la OR & Valor de $\mathrm{p}$ \\
\hline & & & \\
Peldaño, umbral, diferencia de nivel entre habitaciones/espacios & 12,26 & $2,81-53,5$ & $0,001^{\mathrm{a}}$ \\
Deterioro cognitivo y/o demencia & 4,05 & $2,04-8,01$ & $0,001^{\mathrm{a}}$ \\
Accidente cerebrovascular & 2,93 & $1,37-6,28$ & $0,006^{\mathrm{a}}$ \\
Puerta estrecha (menos de $80 \mathrm{~cm}$ de espacio libre) & 2,5 & $1,25-5,04$ & $0,01^{\mathrm{a}}$ \\
Hospitalización en los 2 últimos años & 1,98 & $1,02-3,87$ & $0,044^{\mathrm{a}}$ \\
Enfermedad pulmonar obstructiva crónica & 0,24 & $0,08-0,68$ & $0,008^{\mathrm{a}}$ \\
\end{tabular}

IC 95\%: intervalo de confianza del 95\%; OR: odds ratio.

${ }^{\mathrm{a}} \mathrm{p}<0,05$.

\section{Discusión}

Diversas condiciones de salud, la atención hospitalaria y algunas barreras arquitectónicas influyen en la probabilidad de recibir ayuda personal en los desplazamientos dentro del hogar, en los sujetos dependientes estudiados.

El deterioro cognitivo y la demencia predicen el apoyo de otra persona en la deambulación interior. Este resultado corrobora investigaciones desarrolladas con personas mayores no institucionalizadas, a nivel internacional ${ }^{15}$ y en la población española ${ }^{16 \text { and }}{ }^{17}$. La asociación detectada entre el ACV y la ayuda de un cuidador es consistente con trabajos realizados en otros países ${ }^{15}$ y en España ${ }^{18}$. En ambas condiciones de salud, la aparición de deficiencias en las funciones mentales y/o motrices ocasiona las limitaciones en la actividad y la situación de dependencia, en coherencia con el modelo expuesto en la $\mathrm{CIF}^{2}$. Sin embargo, los sujetos con EPOC se desplazan mayoritariamente sin ayuda, compensando la alteración respiratoria con medidas de conservación de la energía como realizar descansos o el uso de productos de apoyo.

Según otros estudios ${ }^{19}$ and 20 , la hospitalización se asocia con la ayuda recibida en las actividades cotidianas. Esta relación se explica por el perfil de los usuarios de esta modalidad terapéutica, 
caracterizado por la necesidad de cuidados especializados y continuos, no susceptibles de ser prestados en régimen ambulatorio o en el domicilio ${ }^{21}$.

Este trabajo confirma los resultados de investigaciones anteriores realizadas con poblaciones diferentes a la analizada en el presente estudio, en relación con la asociación de las barreras con el apoyo personal. Un diseño longitudinal estableció que los sujetos mayores que residen en hogares con barreras son menos independientes en las actividades diarias ${ }^{3}$. Análisis multivariantes realizados en estudios transversales concluyeron que existe una relación significativa entre la existencia de barreras y la ayuda en el autocuidado. Por ejemplo, utilizar puertas de ancho inadecuado aumenta la OR de recibir ayuda informal en usuarios de silla de ruedas ${ }^{4}$. En sujetos con discapacidad física, la percepción de barreras arquitectónicas (p. ej., escalones o puertas estrechas) se asocia con una mayor probabilidad de experimentar dificultad o recibir apoyo en actividades como la deambulación interior ${ }^{6}$. Otra investigación identificó resultados similares en los meses posteriores a un $\mathrm{ACV}^{5}$.

Los resultados del estudio apoyan el modelo biopsicosocial de la $\mathrm{OMS}^{2}$, caracterizado por destacar la influencia del entorno sobre el funcionamiento, al comprobar que algunas barreras son factores explicativos de la dependencia. La CIF $^{2}$ postula que el ser humano se encuentra en una interacción dinámica y bidireccional con el contexto. Ambos elementos de la relación, persona y entorno, determinan el nivel de funcionamiento. Algunas características del contexto, por ejemplo, los obstáculos en el diseño arquitectónico ocasionan o agravan una discapacidad ${ }^{2}$. La presente investigación demuestra que la existencia de escalones o diferencias de nivel, así como el ancho inadecuado de las puertas, predicen la ayuda personal recibida en los desplazamientos. Con respecto a la primera de las barreras, la asociación alcanza la relevancia clínica, dada la magnitud elevada de la OR.

La CIF $^{2}$ incluye la adaptación domiciliaria dentro de los factores contextuales facilitadores del funcionamiento. Esta intervención, consistente en la supresión de los obstáculos arquitectónicos para las actividades cotidianas, produce un retraso en la progresión de la dependencia, según los resultados obtenidos en un ensayo clínico desarrollado con personas mayores con dificultad en el desempeño diario $^{22}$. En otro diseño experimental, los sujetos que removieron barreras percibían un nivel de seguridad elevado y un grado de dificultad reducido en las tareas ${ }^{23}$.

Para favorecer la permanencia en el hogar, el desempeño independiente, disminuir la necesidad de atención profesional y evitar la «carga» del cuidador, los Servicios de Rehabilitación deben promover la adaptación del hogar mediante el trabajo coordinado del médico rehabilitador y del terapeuta ocupacional. En los servicios sanitarios españoles, esta intervención se desarrolla principalmente en el nivel de Atención Especializada (hospitalización y consultas), siendo necesario incorporar la formación en adaptaciones del hogar dentro de las acciones de educación sanitaria desarrolladas en los centros de Atención Primaria, así como en los diferentes dispositivos de la red de Servicios Sociales (centros comunitarios, de día y asociaciones).

Con respecto a las limitaciones del estudio, el diseño transversal no permite establecer la secuencia temporal de la asociación entre las barreras y la ayuda personal, lo que impide la identificación de la relación causal.

Como conclusiones, la dependencia en los desplazamientos se explica por varias condiciones de salud, la hospitalización y la presencia de algunas barreras. Se confirma la influencia ejercida por el contexto físico domiciliario sobre el funcionamiento, en el desempeño de las actividades y la participación, al comprobar que las barreras arquitectónicas predicen la ayuda personal en la deambulación dentro del hogar. Los Servicios de Rehabilitación deben incorporar el potencial terapéutico de la supresión de las barreras del domicilio, con el objetivo de promover la independencia en el desempeño diario.

\section{Responsabilidades éticas}

Protección de personas y animales

Los autores declaran que para esta investigación no se han realizado experimentos en seres humanos ni en animales.

\section{Confidencialidad de los datos}

Los autores declaran que han seguido los protocolos de su centro de trabajo sobre la publicación de datos de pacientes. El estudio fue revisado y aprobado por el Comité Ético de Investigación Clínica de Galicia y por la Xunta de Galicia. De una forma previa a la realización del estudio, la Xunta de Galicia llevó a cabo una disociación de la información recogida en los BVD, procedimiento definido en la legislación como "el tratamiento de datos de modo que la información que se obtenga no pueda asociarse a persona identificada o identificable" (artículo 3 de la Ley Orgánica 15/1999, de 13 de diciembre, de Protección de 
Datos de Carácter Personal). Los datos disociados no permiten la identificación del afectado o interesado. Mediante la ejecución de este procedimiento, los investigadores recibieron y analizaron la información procedente de copias de BVD previamente disociados por la Administración, por lo que se garantiza la confidencialidad de los datos y se cumple con la Ley Orgánica 15/1999, de 13 de diciembre, de Protección de Datos de Carácter Personal.

\section{Conflicto de intereses}

Los autores declaran no tener ningún conflicto de intereses.

\section{Bibliografía}

1. Instituto de Mayores y Servicios Sociales. Cuidados a las personas mayores en los hogares españoles. El entorno familiar. Instituto de Mayores y Servicios Sociales, Madrid (2005).

2. Organización Mundial de la Salud. Clasificación Internacional del Funcionamiento, de la Discapacidad y de la Salud. Ministerio de Trabajo y Asuntos Sociales, Madrid (2001).

3. S. Iwarsson. A long-term perspective on person-environment fit and ADL dependence among older Swedish adults. Gerontologist, 45 (2005), pp. 327-336.

4. S. Allen, L. Resnik, J. Roy. Promoting independence for wheelchair users: The role of home accommodations. Gerontologist, 46 (2006), pp. 115-123.

5. A. Rochette, J. Desrosiers, L. Noreau. Association between personal and environmental factors and the occurrence of handicap situations following a stroke. Disabil Rehabil, 23 (2001), pp. 559-569.

6. M.G. Stineman, R.N. Ross, G. Maislin, D. Gray. Population-based study of home accessibility features and the activities of daily living: Clinical and policy implications. Disabil Rehabil, 29 (2007), pp. 1165-1175.

7. Real decreto 504/2007, de 20 de abril, por el que se aprueba el baremo de valoración de la situación de dependencia establecido por la Ley 39/2006, de 14 de diciembre, de promoción de la autonomía personal y atención a las personas en situación de dependencia..

8. J. Martínez, R. Dueñas, M.C. Onís, C. Aguado, C. Albert, R. Luque. Adaptación y validación al castellano del cuestionario de Pfeiffer (SPMSQ) para detectar la existencia de deterioro cognitivo en personas mayores de 65 años. Med Clin (Barc), 117 (2001), pp. 129-134.

9. M.E. Charlson, P. Pompei, K.L. Ales, C.R. MacKenzie. A new method of classifying prognostic comorbidity in longitudinal studies: Development and validation. J Chronic Dis, 40 (1987), pp. 373-383.

10. L.F. Berkman, L. Leo-Summers, R.I. Horwitz. Emotional support and survival after myocardial infarction. A prospective, population-based study of the elderly. Ann Intern Med, 117 (1992), pp. 1003-1009.

11. G. Carlsson, O. Schilling, B. Slaug, A. Fänge, A. Ståhl, C. Nygren, et al. Toward a screening tool for housing accessibility problems. J Appl Gerontol, 28 (2009), pp. 59-80.

12. S. Iwarsson, B. Slaug, A.M. Fänge. The housing enabler screening tool: Feasibility and interrater agreement in a real estate company practice context. J Appl Gerontol, 31 (2012), pp. 641-660.

13. S. Iwarsson, M. Haak, B. Slaug. Current developments of the housing enabler methodology. Br J Occup Ther, 75 (2012), pp. 517-521.

14. Decreto 35/2000, de 28 de enero, por el que se aprueba el reglamento de desarrollo y ejecución de la ley de accesibilidad y supresión de barreras en la Comunidad Autónoma de Galicia.

15. T.S. Alexandre, L.P. Corona, D.P. Nunes, J.L.F. Santos, Y.A.O. Duarte, M.L. Lebrão. Gender differences in incidence and determinants of disability in activities of daily living among elderly individuals: SABE study. Arch Gerontol Geriatr, 55 (2012), pp. 431-437.

16. E. Rubio, A. Lázaro, T. Martínez, R. Magallón. Enfermedades crónicas y deterioro funcional para las actividades de la vida diaria en población mayor no institucionalizada. Rev Esp Geriatr Gerontol, 44 (2009), pp. 244-250.

17. E. Sitjas, A. San José, L. Armadans, X. Mundet, M. Vilardell. Factores predictores del deterioro funcional geriátrico. Aten rimaria, 32 (2003), pp. 282-328.

18. F. Formiga, A. Ferrer, J.M. Pérez-Castejón, C. Olmedo, R. Pujol. Risk factors for functional decline in nonagenarians: A one-year follow-up. The NonaSantfeliu study. Gerontology, 53 (2007), pp. 211-217.

19. D. Corrales, L. Palomo, M.J. Magariño, G. Alonso, P. Torrico, A. Barroso, et al. Capacidad funcional y problemas socioasistenciales de los ancianos del área de salud de Cáceres. Aten Primaria, 33 (2004), pp. 426 433.

20. T. Ishizaki, S. Watanabe, T. Suzuki, H. Shibata, H. Haga. Predictors for functional decline among nondisabled older Japanese living in a community during a 3-year follow-up. J Am Geriatr Soc, 48 (2000), pp. 1424-1429.

21. Real Decreto 1030/2006, de 15 de septiembre, por el que se establece la cartera de servicios comunes del Sistema Nacional de Salud y el procedimiento para su actualización..

22. W.C. Mann, K.J. Ottenbacher, L. Fraas, M. Tomita, C.V. Granger. Effectiveness of assistive technology and environmental interventions in maintaining independence and reducing home care costs for the frail elderly. A randomized controlled trial. Arch Fam Med, 8 (1999), pp. 210-217.

23. I. Petersson, M. Lilja, J. Hammel, A. Kottorp. Impact of home modification services on ability in everyday life for people ageing with disabilities. J Rehabil Med, 40 (2008), pp. 253-260. 